\title{
Espectroscopia no infravermelho próximo para estimativa da densidade básica de madeiras de Pinus
}

\author{
Marcelo Lazzarotto $^{1 \star}$, Robson Ribeiro Netipanyj ${ }^{2}$, Washington Luiz Esteves Magalhães ${ }^{1}$, Ananda Vir- \\ ginia De Aguiar ${ }^{1}$ \\ ${ }^{1}$ Embrapa Florestas, Estrada da Ribeira, Km 111 - Bairro Guaraituba - Caixa Postal: 319 - CEP: 83411-000 - Colombo -PR, \\ Brasil. \\ ${ }^{2}$ Pontifícia Universidade Católica do Paraná, Rua. Imaculada Conceição, 1155 - CEP 80215-901- Prado Velho, Curitiba - PR, \\ Brasil.
}

\begin{abstract}
RESUMO A densidade básica é uma das mais importantes características das madeiras e usada em programas de melhoramento genético florestal. Este trabalho teve o objetivo de construir um modelo de predição da densidade básica de madeiras de Pinus maximinoi e Pinus tecunumanii, usando a espectroscopia no infravermelho próximo e análise estatística multivariada. O modelo foi construído com 54 amostras usando mínimos múltiplos quadrados (PLS) como tratamento estatístico (com alisamento do sinal, variação normal padrão SNV e primeira derivada). A curva de calibração apresentou coeficiente de determinação $\left(\mathrm{R}^{2}\right)$ de 0,94 e erro de validação cruzada de aproximadamente $30 \mathrm{~kg} \mathrm{~m}^{-3}$ para uma densidade básica média de $517 \mathrm{~kg} \mathrm{~m}^{-3}$. Este modelo foi validado externamente com 27 amostras não usadas na construção da curva de calibração. $O$ erro da validação externa foi de aproximadamente $47 \mathrm{~kg} \mathrm{~m}^{-3} \mathrm{e}$ a razão de desempenho do desvio (RPD) de 1,9, mostrando que o modelo pode ser usado para seleção prévia de grande número de amostras.
\end{abstract}

Palavras-chave: NIR; quimiometria, densidade básica; regressão PLS; qualidade da madeira.

\section{Near infrared spectroscopy to estimate the basic density of Pinus woods}

\begin{abstract}
The basic density is one of the most important characteristics of wood and is used in forest breeding programs. This work aims to develop a prediction model for the basic density of Pinus maximinoi and Pinus tecunumanii woods using near infrared spectroscopy and multivariate statistical analysis. The model was developed with 54 samples using partial least square (PLS) as statistical treatment (with signal smoothing, Standard Normal Variate SNV and first derivative). The calibration curve showed a coefficient of determination $\left(\mathrm{R}^{2}\right)$ of 0.94 and error of cross validation of approximately $30 \mathrm{~kg} \mathrm{~m}^{-3}$ for a basic density average of $517 \mathrm{~kg} \mathrm{~m}^{-3}$. This model was external validated with 27 samples that were not used in the construction of the calibration curve. The error of the external validation was approximately $47 \mathrm{~kg} \mathrm{~m}^{-3}$ and the ratio of performance to deviation (RPD) of 1.9, showing that this model can be used for screening selection of a great number of samples.
\end{abstract}

Keywords: NIR; chemometrics; basic density; PLS regression; wood quality.

\section{Introdução}

Para o melhoramento genético de espécies florestais são necessárias caracterizações de um grande número de amostras. Por este motivo, as técnicas de análises rápidas são muito utilizadas para o desenvolvimento de novos materiais genéticos. Por meio desses melhoramentos genéticos, é possível a criação de clones visando a padronização da qualidade da madeira, a qual é uma exigência industrial cada vez maior (LAZZAROTTO; MAGALHÃES, 2014; ISIK et al., 2011; CAPPA at al., 2013; GRATTAPAGLIA; RESENDE, 2011).

A densidade básica é uma das características mais importantes da madeira, pois apresenta relação com muitas outras 
propriedades. Uma das melhores correlações é com as propriedades mecânicas, mas existem trabalhos correlacionando-a com o teor de lignina, a razão cerne e alburno, entre outras (DIAS; LAHR, 2004; LOBÃO, 2004; BARRICHELO; BRITO, 1979).

Em muitas atividades de laboratório, consideram-se como técnicas não destrutivas as que não necessitam destruir a amostra, caracterizando-a quantas vezes se desejar. No setor florestal, o método de análise mais interessante seria aquele em que não fosse necessário o abate da árvore e as propriedades de interesse pudessem ser caracterizadas de maneira muito rápida e com baixo custo. Todavia, é também considerado como método não-destrutivo aquele em que são retirados rolos de incremento à altura do peito (DAP) e que apenas estas pequenas amostras são destruídas no laboratório.

A medida não destrutiva da densidade básica da madeira pode ser feita por meio de tomografia de raios-X ou gama. No entanto, a técnica é morosa e exige um operador treinado e autorizado a operar fontes radioativas (MACEDO et al., 2002).

Outra possibilidade é o emprego de ondas sonoras, entretanto, a velocidade de propagação da onda de som ou de ultrassom não depende apenas da densidade básica, mas também do módulo de elasticidade da madeira. Dessa maneira, a técnica apresenta uma baixa correlação com a densidade básica, a menos que o módulo de elasticidade seja conhecido, o que inviabiliza a precisão da técnica (BAAR, J. et al., 2012). No entanto, esta técnica permite caracterizar a madeira de uma árvore sem a necessidade de remoção de rolo de incremento, com rapidez e baixo custo.

Equipamentos como o Pilodyn ou o resistógrafo também podem ser empregados e foram avaliados por Gouvêa et al. (2011). A prática mostra a grande imprecisão dessas medidas e a baixa correlação entre a densidade básica e a penetração da agulha de aço do Pilodyn e a resistência da broca do resistógrafo. A broca do resistógrafo perde o fio de sua amolação com o tempo de uso, deixando a técnica menos confiável com o passar do tempo (LIMA et al, 2007; CHARRIA et al., 2014;). Por outro lado, esses métodos também apresentam muitas vantagens, pois não necessitam que as amostras sejam retiradas, além da fácil operação e baixo custo.

Outra técnica que tem se mostrado capaz de determinar a densidade básica da madeira é a espectroscopia na região do infravermelho próximo - NIRS (LAZZAROTTO; MAGALHÃES, 2014; CARNEIRO et al., 2010; MAGALHÃES et al., 2006). A técnica de espectroscopia NIR utiliza a faixa do espectro eletromagnético entre 800 e $2500 \mathrm{~nm}$. Nessa região de comprimento de onda (l) os grupos funcionais contendo carbono, hidrogênio, oxigênio e nitrogênio absorvem radiação incidente, transformando a energia absorvida em vibrações desses grupos. A energia da luz incidente que não for absorvida é parcialmente transmitida e refletida. A luz refletida difusamente, ou seja, que não seja da reflexão especular, é medida em detectores do equipamento e por diferença calculase quanto de intensidade de luz foi absorvida pela amostra. Cada grupo funcional absorve em um comprimento de onda (ou a frequência $=$ velocidade da luz/ l) típico, e a intensidade da luz absorvida é proporcional à concentração do grupo absorvedor. Informações sobre características físicas e químicas do material podem ser inferidas a partir da quantidade de luz absorvida e do comprimento de onda. O NIR tem uma absorção entre 10 e 100 vezes menor que infravermelho médio, o que confere a vantagem de permitir medidas com quase nenhum tratamento prévio da amostra. Estudos com essa técnica vêm se difundindo como método de análise devido à sua rapidez, a pouca preparação de amostras, nenhum uso de reagentes químicos e nenhuma influência do operador (HEIN et al., 2016; HWANG et al., 2016; PEREIRA et al., 2016; TSUCHIKAWA; KOBORI, 2015). 
O emprego da espectroscopia na região do NIR pode ser usado para a estimativa da densidade básica da madeira com diversas formas de amostragem. Carneiro et al. (2010) correlacionaram a densidade básica com espectros de lâminas de madeira, e Magalhães et al. (2006) obtiveram curvas de calibração com alta correlação da densidade básica em cubos de madeira.

Esse trabalho objetiva estimar a densidade básica da madeira de Pinus maximinoi e Pinus tecunumanii por meio da construção de um modelo multivariado a partir de espectros na região do NIR obtidos diretamente de rolos de incremento.

\section{Material e Métodos}

\section{Amostragem e preparação do material}

Foram utilizadas amostras de madeira de $P$. maximinoi e P. Tecunumanii coletadas de 32 árvores de cada espécie, totalizando 64 indivíduos. De cada uma delas foram coletados rolos de incremento (Figura 1) por meio de uma sonda Pressler. Os orifícios de coleta foram feitos à altura do peito (DAP). Os rolos de incremento foram divididos em três partes no sentido medula-casca, totalizando 192 amostras. Selecionaram-se 102 amostras, de maneira a abranger o máximo de variação de densidade básica. Análise estatística dos espectros dos rolos de incremento mostrou a presença de 21 amostras discrepantes, provavelmente pelo formato cilíndrico irregular e oxidação dessas amostras causadas pela ferramenta de extração. Assim, para a elaboração do modelo foram usadas 41 amostras de $P$. maximinoi e 40 amostras de P. Tecunumanii, totalizando 81 amostras.

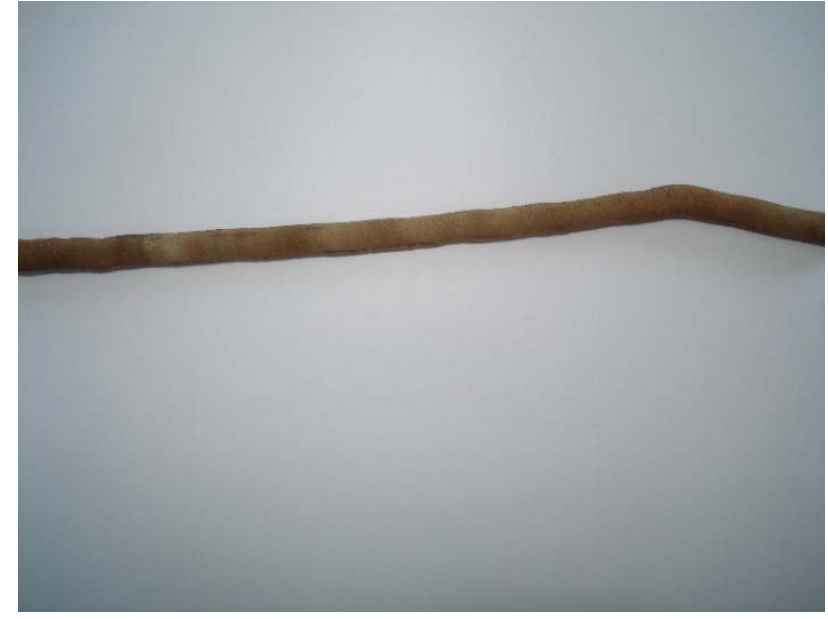

Figura 1. Exemplo do rolo de incremento usado para determinação da densidade básica das amostras e aquisição dos espectros de NIR.

Figure 1. Example of the increment core used for the determination of the basic density of the samples and the acquisition of the NIR spectra.

\section{Determinação da densidade básica da madeira}

A densidade básica foi determinada pelo método gravimétrico. As amostras foram imersas em água durante sete dias para saturação total. Foram determinados os pesos imersos das amostras saturadas com o auxílio de um dispositivo especial acoplado ao prato da balança. Posteriormente, foi realizada a pesagem das amostras após a remoção da água superficial em excesso, obtendo-se o peso saturado. Depois foi realizada secagem das amostras em estufa a $105 \pm 3^{\circ} \mathrm{C}$ até peso constante para a obtenção do peso seco. A balança usada tem precisão na terceira casa decimal $(0,001 \mathrm{~g})$, portanto o erro da medida convencional da densidade básica é de no máximo de $1 \mathrm{~kg} \mathrm{~m}^{-3}$. A densidade básica foi calculada utilizando-se a Equação 1:

$$
\rho \mathrm{b}=\mathrm{PS} /(\text { PSAT-PI) } \quad \text { Equação } 1
$$

Onde: $\rho b=$ densidade básica $\left(\mathrm{kg} \mathrm{m}^{-3}\right)$; PS = peso seco $(\mathrm{g})$; PSAT $=$ peso saturado $(\mathrm{g}) ; \mathrm{PI}=$ peso imerso $(\mathrm{g})$. 


\section{Aquisição dos espectros de NIR}

Os espectros na região do NIR foram obtidos usando o equipamento Bruker Tensor 37 a partir de pequenas porções dos rolos de incremento secos em estufa $\left(105 \pm 3^{\circ} \mathrm{C}\right)$ após a medida convencional da densidade básica. Os espectros foram coletados através de 64 varreduras espectrais para cada amostra, com uma resolução de $2 \mathrm{~cm}^{-1}$ entre os números de onda de 4.000 a $10.000 \mathrm{~cm}^{-1}$, no modo de reflectância difusa. Os espectros de reflectância foram utilizados nas análises quimiométricas após a transformação da porcentagem de reflectância em absorbância [log10 (1/Reflectância)].

\section{Modelos de calibração}

Para a construção do modelo de calibração foram usados as amostras de $P$. maximinoi e $P$. tecunumanii na regressão dos Mínimos Quadrados Parciais (PLS) utilizando o programa Unscrambler ${ }^{\oplus}$ (CAMO AS, Norway) versão 10.3. Visando remover os ruídos dos espectros foram aplicados diferentes tratamentos matemáticos no espectro. As operações simultâneas de alisamento, SNV e primeira derivada nos espectros foram as mais adequadas. O modelo foi obtido usando os tratamentos Alisamento (polinomial de segunda ordem e 10 pontos de cada lado), SNV e Primeira Derivada (polinomial de segunda ordem e 10 pontos de cada lado).

O grupo de dados foi dividido em duas partes, 2/3 foram usados para a construção do modelo de calibração (54 amostras) e o $1 / 3$ restante para a validação externa (27 amostras). A seleção das amostras para o subgrupo de calibração foi realizada organizando em ordem ascendente das medidas de referência, e selecionando as amostras para que estivessem uniformemente distribuídas. A calibração foi ajustada com o número máximo de 12 variáveis latente. $\mathrm{O}$ subgrupo de validação foi construído usando as amostras complementares. Para estimar a qualidade dos modelos de calibração foram usados os resultados de RPD, Razão da Performance do Desvio, Equação 2. Esse procedimento permite alto controle da variabilidade em cada subgrupo.

$$
\mathrm{RPD}=\mathrm{SD} / \mathrm{SE} \quad \text { Equação } 2
$$

Onde: $\mathrm{RPD}=$ razão do desvio de performance; $\mathrm{SD}=$ desvio padrão da amostra na análise convencional; SE = erro de predição.

\section{Resultados e Discussão}

Os espectros na região do NIR são típicos de madeira (Figura 2) e com o mesmo padrão dos espectros obtidos por Jones et al. (2005).

A Figura 3 apresenta os coeficientes de regressão em função dos comprimentos de onda, usando-se oito variáveis latentes. Para a obtenção da densidade básica basta realizar a soma algébrica dos coeficientes de predição multiplicados pelas respectivas absorbâncias após as operações de alisamento, SNV e primeira derivada do espectro. Neste modelo, o valor do $\mathrm{B}_{0}$ é de $790 \mathrm{~kg} \mathrm{~m}^{-3}$. As regiões destacadas correspondem aos comprimentos de onda do espectro de infravermelho estatisticamente significativos e as grandezas das barras são as contribuições para construção do modelo PLS.

A curva de calibração construída usando regressão PLS apresentou coeficiente de determinação $\left(R^{2}\right)$ de 0,94 e o erro de validação cruzada foi de $30 \mathrm{~kg} \mathrm{~m}^{-3}$ para uma densidade básica média de $517 \mathrm{~kg} \mathrm{~m}^{-3}$. Lazzarotto; Magalhães (2014) em estudo usando o NIR para estimar a densidade básica de madeira de Eucalyptus spp. obtiveram valores de $\mathrm{R}^{2}=0,83$ e o erro de validação cruzada de $45 \mathrm{~kg} \mathrm{~m}^{-3}$ para uma densidade básica média de $467 \mathrm{~kg} \mathrm{~m}^{-3}$. As vibrações mais importantes para a estimativa da densidade básica (Figura 3) de $P$. maximinoi e $P$. tecunumanii estão relacionadas com as 
principais ligações dos grupos funcionais da celulose, das hemiceluloses e da lignina (Figura 2).

A Figura 4 mostra os valores de referência medidos em laboratório de forma convencional para a densidade básica correlacionados com os valores calculados com base nos espectros de NIR obtidos da madeira.

A curva de calibração obtida foi testada com amostras que não participaram na construção da curva de calibração (predição externa). O erro padrão de predição encontrado foi de $46,8 \mathrm{~kg} \mathrm{~m}^{-3}$, com um coeficiente de determinação $\mathrm{R}^{2}$ de 0,73, sendo o número de amostras usadas nessa validação externa igual a 27.

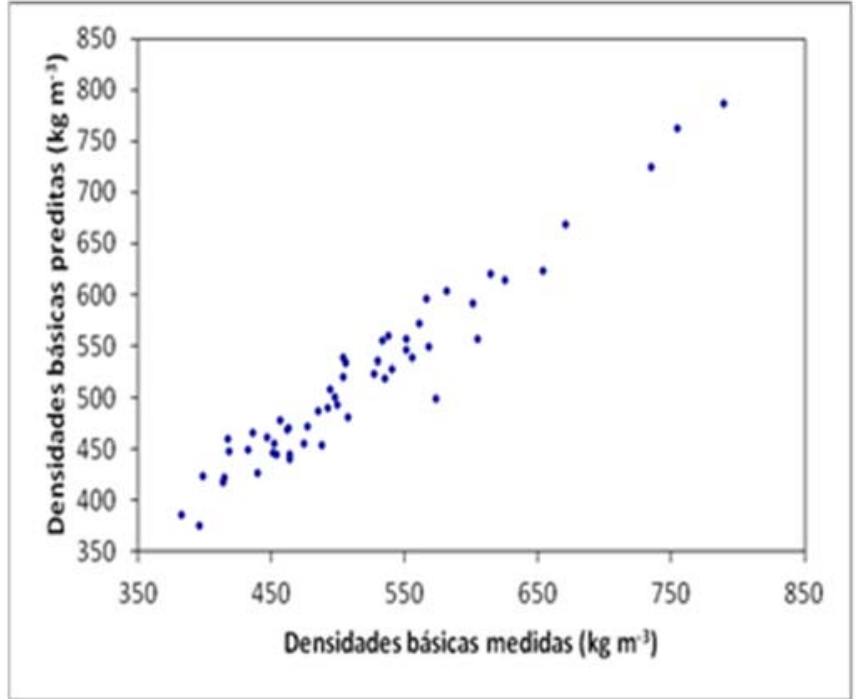

Figura 4. Relação entre os valores de densidade básica medidos pelo método convencional e estimados por NIR.

Figure 4. Relationship between basic density values measured by the conventional method and estimated by NIR.

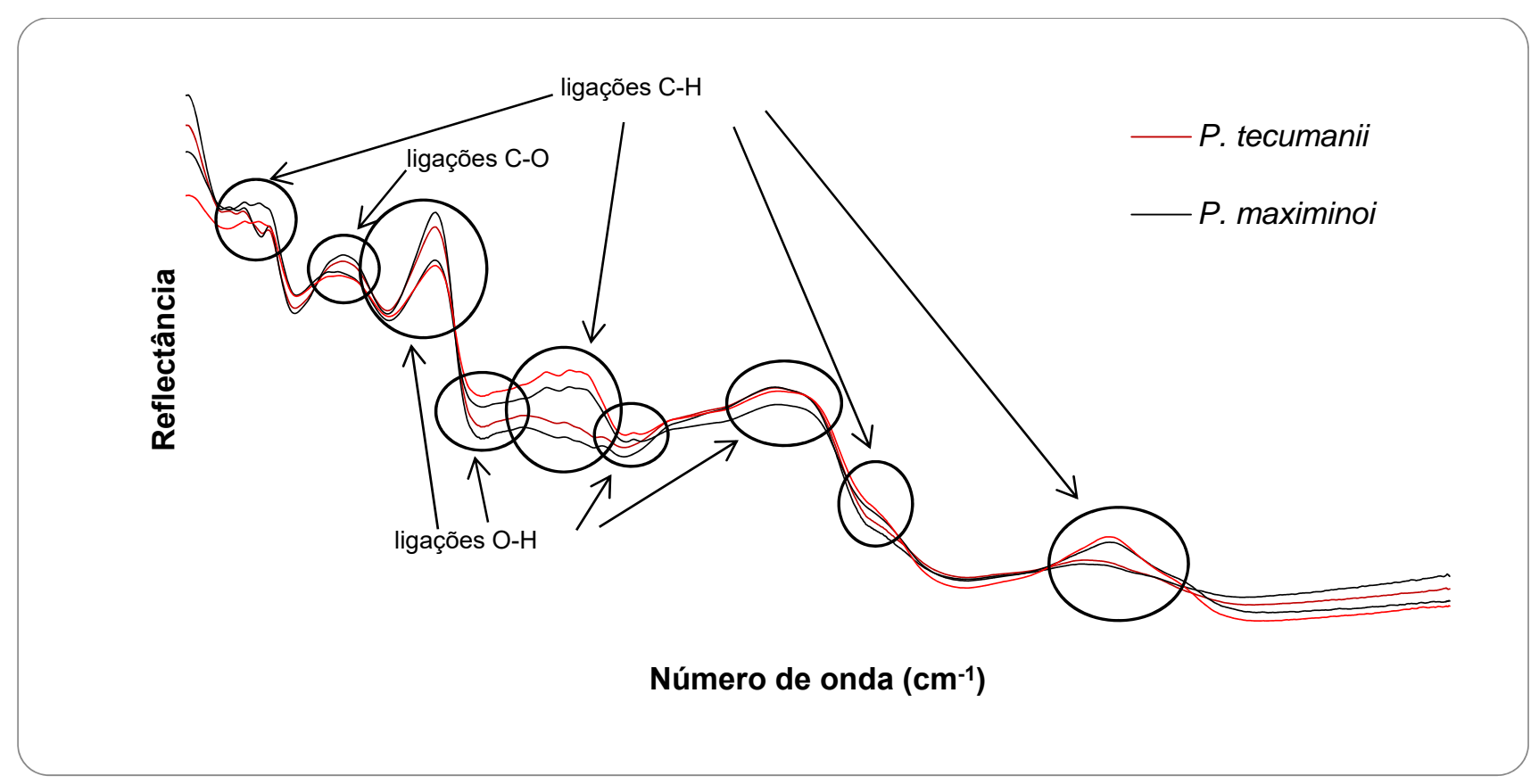

Figura 2. Espectros típicos de madeira obtidos para quatro amostras após transformações de alisamento e SNV. As regiões destacadas referem-se as principais ligações dos grupos funcionais presentes nos constituintes da madeira (celulose, hemiceluloses e lignina).

Figure 2. Typical spectra for wood obtained from four samples after smoothing and SNV transformations. Highlighted regions refer to the main linkages of the functional groups of wood constituents (cellulose, hemicelluloses and lignin). 


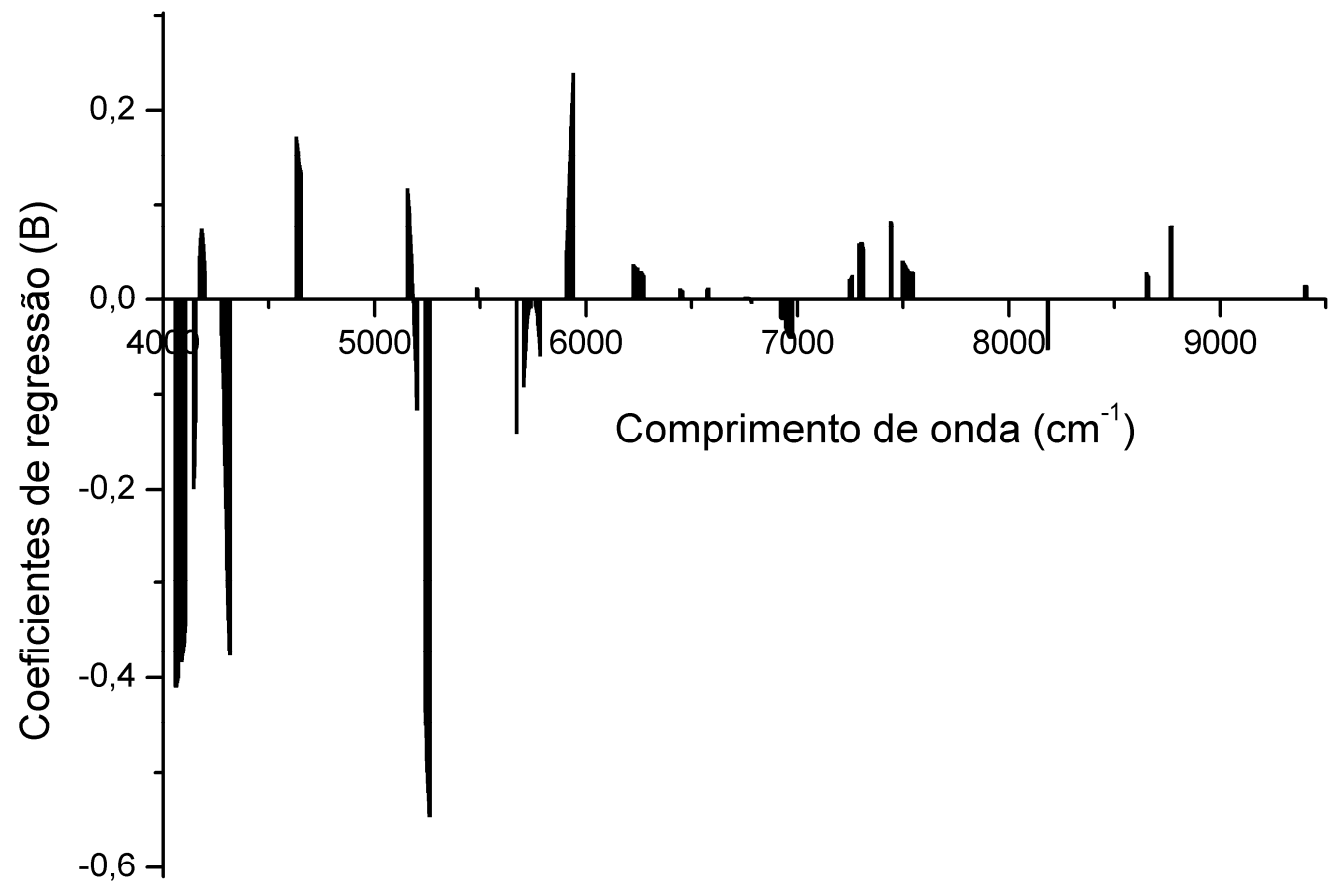

Figura 3. Coeficientes de regressão (B) obtidos no modelo PLS para estimativa da densidade básica usando os espectros de NIR. Figure 3. Regression coefficients (B) obtained in the PLS model to estimate the basic density (B) using the NIR spectra.

A Figura 5 mostra os valores de densidade básica determinados pela análise convencional correlacionados com os valores preditos pela técnica NIR para a validação externa do modelo construído. Os principais parâmetros do modelo de calibração usando PLS e da sua validação externa estão sumarizados na Tabela 1.

A avaliação da qualidade de ajuste da curva de predição pode ser inferida, dentre outros parâmetros, pela razão do desvio de performance (Equação 2).Em estudo anterior visando estimar a densidade básica de madeira de Eucalyptus spp., Lazzarotto; Magalhães (2014) obtiveram os valores de RPD de 2,500, para o grupo de calibração, e de 1,667, para o grupo de predição externa. Esta razão avalia o quanto a técnica pode distinguir entre as amostras diferentes. Altos valores da razão SD/SE (acima de 1,5) indicam que o erro de estimativa de densidade básica é muito menor que o desvio padrão das amostras (SCHIMLECK et al., 2003). Esta avaliação da qualidade do modelo foi inicialmente usada por Williams (1987) sugerindo que valores de RPD maiores do que 3 são usáveis para triagem, valores maiores do que 5 podem ser usados para controle de qualidade e valores maiores do que 8 para qualquer aplicação. Para amostras complexas podem ser usados modelos com valores de RPD acima de 1,5 para uma triagem preliminar (TODOROVIC et al., 2015; ZHOU et al., 2015; PRADES et al., 2014).

Para a construção de um modelo de predição é necessário que o conjunto amostral contemple uma variação muito ampla de valores para a densidade básica. Ressalta-se que a equação de predição deve ser usada dentro da faixa de valores das densidades básicas testadas. 


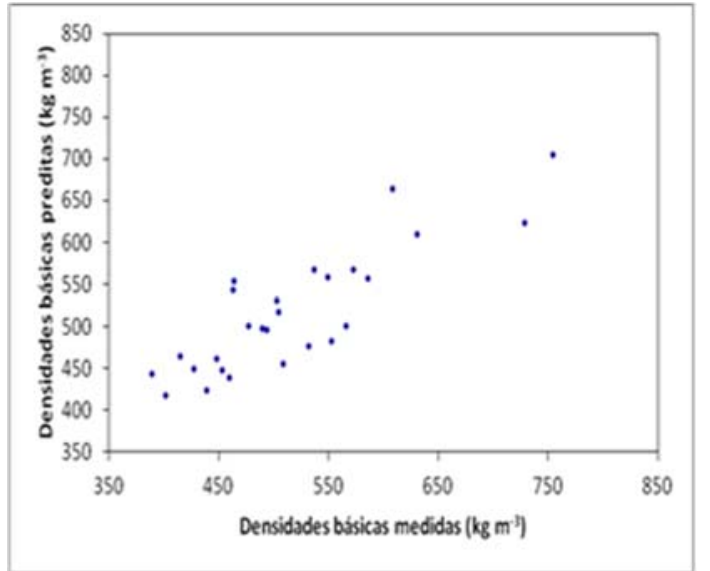

Figura 5. Relação entre os valores de densidade básica medida pelo método convencional e estimados através da espectroscopia no Infravermelho Próximo (NIR)

Figure 5. Relationship between basic density values measured by the conventional method and predicted by NIR.

Tabela 1. Resultados das análises estatísticas para os grupos de calibração e estimativa para os valores de DAP usando o modelo PLS.

Table 1. Results of statistical analysis for calibration and prediction groups for DBH values using the PLS model.

\begin{tabular}{ccc}
\hline Parâmetros & $\begin{array}{c}\text { Grupo de } \\
\text { calibração }\end{array}$ & $\begin{array}{c}\text { Grupo de predição } \\
\text { externa }\end{array}$ \\
\hline Mínimo $\left(\mathrm{kg} \mathrm{m}^{-3}\right)$ & 382,3 & 390,1 \\
Máximo $\left(\mathrm{kg} \mathrm{m}^{-3}\right)$ & 789,2 & 754,7 \\
Média $\left(\mathrm{kg} \mathrm{m}^{-3}\right)$ & 517,5 & 516,8 \\
$\mathrm{SD}\left(\mathrm{kg} \mathrm{m}^{-3}\right)$ & 89,5 & 90,1 \\
$\mathrm{R}^{2}$ & 0,94 & 0,73 \\
$\mathrm{RMSE}\left(\mathrm{kg} \mathrm{m}^{-3}\right)$ & 21,3 & 45,9 \\
$\mathrm{SE}\left(\mathrm{kg} \mathrm{m}^{-3}\right)$ & 21,5 & 46,8 \\
$\mathrm{SD} / \mathrm{SE}$ & 4,16 & 1,93 \\
\hline
\end{tabular}

\section{Conclusões}

Foi possível construir modelos multivariados para predição de densidade básica usando-se quimiometria e PLS (com tratamentos de alisamento, SNV e primeira derivada), e espectrometria na região do infravermelho próximo. Como foi construído um único modelo empregando-se amostras de madeira de $P$. maximinoi e $P$. tecunumanii, uma única curva de calibração pode estimar a densidade básica de madeira de ambas as espécies.

\section{Agradecimentos}

Os autores agradecem a Embrapa (Empresa Brasileira de Pesquisa Agropecuária), ao $\mathrm{CNPq}$ (Conselho Nacional de Pesquisa e Desenvolvimento) e a CAPES (Coordenadoria de Aperfeiçoamento de Pessoal de Nível Superior) pelo suporte dado ao trabalho.

\section{Referências}

BARRICHELO, L. E. G.; BRITO, J. O. Variabilidade radial da madeira de Pinus caribaea var. hondurensis. IPEF, n. 18, p. 81-102, 1979.

BAAR, J.; TIPPNER, J.; GRYC, V. The influence of wood density on longitudinal wave velocity determined by the ultrasound method in comparison to the resonance longitudinal method. European Journal of Wood and Wood Products, v. 70, p. 767-769, 2012. http://dx.doi.org/10.1007/s00107-0110550-2

CAPPA, E. P.; EL-KASSABY, Y. A.; GARCIA, M. N.; ACUNA, C.; BORRALHO, N. M. G.; GRATAPAGLIA, D.; POLTRI, S. N. M. Impacts of population structure and analytical models in genome-wide association studies of complex traits in forest trees: a case study in Eucalyptus globulus, PLoS ONE, 8(11), e81267, 2013.

CARNEIRO, M. E.; MAGALHÃES, W. L. E.; DE MUNIZ, G. I. B.; SCHIMLECK, L. R. Near infrared spectroscopy and chemometrics for predicting specific gravity and flexural modulus of elasticity of Pinus spp. veneers. Journal of Near Infrared Spectroscopy. v. 18, ed 6, p. 481-489, 2010. http://dx.doi.org/10.1255/jnirs.911

CHARRIA, D. L. G.; RODRIGUEZ, V. M. N.; SARMIENTO, M.; BORRALHO, N. Estimación indirecta de la densidad de la madera mediante rl uso de pilodyn em la selección de clones de Eucapyptus pellita F. Muell. Colômbia Florestal, v. 17, n. 2, p. 181-192, 2014. http://dx.doi.org/10.14483/udistrital.jour.colomb.for.2014.2.a04

DIAS, F. M.; LAHR, F. A. R. Estimativa de propriedades de resistência e rigidez da madeira através da densidade aparente. Scientia Florestalis, n. 65, p. 102-113, 2004.

GOUVÊA, A. F. G.; TRUGILHO, P. F.; GOMIDE, J. L.; DA SILVA, J. R. M.; ANDRADE, C. R.; ALVES, I. C. N. Determi- 
nação da densidade básica das madeiras de Eucalyptus por diferentes métodos não destrutivos. Revista Árvore, v. 35, n. 2, p. 349-358, 2011.

GRATTAPAGLIA, D.; RESENDE M. D. V. Genomic selection in forest tree breeding. Tree Genetics \&Genomes, v. 7, p.241-255, 2011. http://dx.doi.org/10.1007/s11295-010$0328-4$

HEIN, P. R. G.; CHAIX, G.; CLAIR, B.; BRANCHERIAU, L.; GRIL, J. Spatial variation of wood density, stiffness and microfibril angle along Eucalyptus trunks grown under contrasting growth conditions. Trees, v. 30, p. 871-882, 2016. http://dx.doi.org/10.1007/s00468-015-1327-8

HWANG, S. W.; HORIKAWA, Y.; LEE, W.; SUGIYAMA, J.; Identification of Pinus species related to historic architecture in Korea using NIR chemometric approaches. Journal of Wood Science, v. 62, p. 156-167, 2016. http://dx.doi.org/10.1007/s10086-016-1540-0

ISIK, F.; MORA, C. R.; SCHIMLECK, L. R. Genetic variation in Pinus taeda wood properties predicted using non-destructive techniques. Annals of Forest Science, v. 68, ed. 2, p.283293, 2011. http://dx.doi.org/10.1007/s13595-011-0035-9

JONES, P. D.; SCHIMLECK, L. R.; PETER, G. F.; DANIELS, R. F.; CLARK, A. Non-destructive estimation of Pinus taeda $\mathrm{L}$ tracheid morphological characteristics for samples from a wide range of sites in Georgia. Wood Science and Technology. v. 39, ed. 7, p. 529-545, 2005. http://dx.doi.org/10.1007/s00226-005-0021-1

LAZZAROTTO, M.; MAGALHÃES, W. L. E. Uso da espectroscopia no infravermelho próximo (NIR) para predição não-destrutiva de densidade básica da madeira de Eucaliptus benthammi e Eucaliptus pellita. Colombo: Embrapa Florestas, 2014. 6 p. (Embrapa Florestas. Comunicado técnico, 334)

LIMA, J. T.; SARTÓRIO, R. C.; TRUGILHO, P. F.; CRUZ, C. R.; VIEIRA, R. S. Uso do resistógrafo para estimar a densidade básica e a resistência à perfuração da madeira de $E u$ calyptus. Scientia Florestalis, n. 75, p. 85-93, 2007.

LOBÃO, M. S.; LÚCIA, R. M. D.; MOREIRA, M. S. S.; GOMES, A. Caracterização das propriedades físico-mecânicas da madeira de eucalipto com diferentes densidades. Revista Árvore, v. 28, n. 6, p. 889-894, 2004.

MACEDO, A.; VAZ, C. M. P.; PEREIRA, J. C. D.; NAIME, J. M.; CRUVINEL, P. E.; CRESTANA, S. Wood density determination by $\mathrm{X}^{-}$and gamma-ray tomography.
Holzforschung, v. 56, ed 5, p 535-540, 2002. http://dx.doi.org/10.1515/HF.2002.082

MAGALHÃES, W. L. E.; ZANGISKI, F.; KLEIN, C. H.; HIGA, A. R. Uso da espectroscopia no infravermelho próximo (NIR) para predição não-destrutiva de densidade básica da madeira de Pinus taeda. Colombo: Embrapa Florestas, 2006. 4 p. (Embrapa Florestas. Comunicado técnico, 159).

PEREIRA, H.; SANTOS, A. J. A.; ANJOS, O. Fibre Morphological Characteristics of Kraft Pulps of Acacia melanoxylon Estimated by NIR-PLS-R Models. Materials, v. 9, n. 8, p. 1-9, 2016. http://dx.doi.org/10.3390/ma9010008

PRADES, C.; GOMEZ-SANCHEZ, I.; GARCIA-OLMO, J.; GONZALEZ-HERNANDEZ, F.; GONZALEZ-ADRADOS, J. R. Application of VIS/NIR spectroscopy for estimating chemical, physical and mechanical properties of cork stoppers. Wood Science and Technology, v. 48, p. 811-830, 2014. http://dx.doi.org/10.1007/s00226-014-0642-3

SCHIMLECK, L. R.; DORAN, J. C.; RIMBAWANTO, A. Near infrared spectroscopy for cost-effective screening of foliar oil characteristics in a Melaleuca cajuputi breeding population. Journal of Agricultural and Food Chemistry, v. 51, p. 2433-2437, 2003. http://dx.doi.org/10.1021/jf020981u

TODOROVIC, N.; POPOVIC, Z.; MILIC, G. Estimation of quality of thermally modified beech wood with red heartwood by FT-NIR spectroscopy. Wood Science and Technology, v. 49 p. 527-549, 2015. http://dx.doi.org/10.1007/s00226-015-0710-3

TSUCHIKAWA, S.; KOBORI, H. A review of recent application of near infrared spectroscopy to wood science and technology. Journal of Wood Science, v. 61, p. 213-220, 2015. http://dx.doi.org/10.1007/s10086-015-1467-x

Williams, P.C. 1987. Variables affecting nearinfraredreflectance spectroscopic analysis. In: Near- Infrared Technology in the Agricultural and Food Industries (eds P. Williams \& K. Norris), pp. 143 - 167. American Association of Cereal Chemists Inc. Saint Paul, MN.

ZHOU, C.; JIANG, W.; VIA, B. K.; FASINA, O.; HAN, G. T. Prediction of mixed hardwood lignin and carbohydrate content using ATR-FTIR and FT-NIR. Carbohydrate Polymers, v. 121, p. 336-341, 2015. http://dx.doi.org/10.1016/j.carbpol.2014.11.062 